\title{
Accelerated partial breast irradiation with brachytherapy: patient selection and technique considerations
}

This article was published in the following Dove Press journal:

Breast Cancer: Targets and Therapy

29 July 2015

Number of times this article has been viewed

\author{
Daniel M Trifiletti' \\ Kara D Romano' \\ Shayna L Showalter ${ }^{2}$ \\ Kelli A Reardon' \\ Bruce Libby' \\ Timothy N Showalter' \\ 'Department of Radiation Oncology, \\ ${ }^{2}$ Division of Surgical Oncology, \\ Department of Surgery, University \\ of Virginia School of Medicine, \\ Charlottesville, VA, USA
}

\begin{abstract}
Accelerated partial breast irradiation (APBI) through breast brachytherapy is a relatively recent development in breast radiotherapy that has gained international favor because of its reduction in treatment duration and normal tissue irradiation while maintaining favorable cancer-specific and cosmetic outcomes. Despite the fact that several large national trials have not reported final results yet, many providers are currently offering APBI to select patients and APBI is listed as a treatment option for selecting patients in the National Comprehensive Cancer Network guidelines. Multiple consensus guidelines exist in selecting patients for APBI, some with conflicting recommendations. In this review, the existing patient selection guidelines are reported, compared, and critiqued, grouping them in helpful subcategories. Unique patient and technical selection factors for APBI with brachytherapy are explored.
\end{abstract}

Keywords: breast cancer, APBI, breast brachytherapy

\section{Introduction}

In most patients with early stage breast cancer, adjuvant radiotherapy (RT) is part of a standard of care approach for breast conserving therapy (BCT). ${ }^{1}$ Although BCT was originally validated utilizing whole breast irradiation (WBI) over a 5- to 6.5-week course, ${ }^{2}$ there has since been an international trend aimed at evaluating a reduced number of RT treatments (hypofractionated or accelerated RT) and a reduction in the volume of breast irradiated (partial breast RT). A combination of these two approaches, termed accelerated partial breast irradiation (APBI), is currently being investigated in several large national trials with results pending. ${ }^{3}$ Despite the lack of this mature phase III data, preliminary results suggest that APBI is safe and effective. ${ }^{4}$ As a result, several consensus groups including the National Comprehensive Cancer Network endorse the use of APBI in selecting patients. ${ }^{1,4-7}$

Brachytherapy, an RT technique that places radioactive isotopes within or adjacent to a tumor or tumor bed, has been utilized in cancer care for over a century. ${ }^{8}$ It has the benefit of ultra-high radiation dose delivery to the tissue adjacent to the isotope but a rapid fall-off of radiation dose as distance from the source increases. It is this characteristic of brachytherapy that has made it an ideal choice for APBI: it can be used to treat the lumpectomy cavity to high radiation doses while limiting radiation exposure to the non-involved ipsilateral breast, skin, lung, heart, and contralateral breast. For this reason, the use of brachytherapy to treat breast cancer has gained significant popularity in the last 10-20 years and APBI using brachytherapy devices was included as a technique in the pending international trials of APBI. ${ }^{3}$
Correspondence: Timothy N Showalter Department of Radiation Oncology, University of Virginia School of Medicine, 1240 Lee Street, Box 800383 , Charlottesville, VA 22908, USA

Tel +l 4349826278

Fax +l 4349823262

Email tns3b@virginia.edu 
Significant controversy exists in the definition of the "select patients" for whom APBI should be endorsed as a safe and effective option and consensus statements from multiple medical and surgical societies provide conflicting definitions of this group of patients (Table 1) $\cdot{ }^{4-7}$ Moreover, an estimated $60 \%$ of patients treated with brachytherapy-based APBI nationally would be considered as "cautionary" or "unsuitable" by consensus expert guidelines. ${ }^{9}$ The purpose of this article is to organize and synthesize the evidence supporting current consensus guidelines for APBI and focus on unique clinical scenarios that could alter breast brachytherapy APBI management. This report is a narrative review of the literature, comprising a search of PubMed and the authors' knowledge of available citations, and does not constitute a formal systematic review or meta-analysis.

\section{Recommending APBI as a part of a BCT approach}

The rationale for APBI is based on the fact that $90 \%$ of ipsilateral breast tumor recurrences (IBTR) occur within $1 \mathrm{~cm}$ of the lumpectomy cavity (defined here as local IBTR). ${ }^{10}$ The goal of patient selection for APBI is to define a group of woman that are at risk for local failure and would benefit from adjuvant RT, but are at sufficiently low risk for regional IBTR (defined here as more than $1 \mathrm{~cm}$ from the lumpectomy cavity) and sufficiently low risk for nodal and distant failure. It is for these women that APBI is both beneficial and sufficient.

\section{Factors predicting for local IBTR}

Tumor and patient characteristics that serve as predictors of local failure have been identified by the long-term followup of several randomized clinical trials. ${ }^{11-14}$ Well-validated factors predictive of increased risk of local failure include: younger age at diagnosis, involved margins, larger size, higher grade, lymph vascular space invasion (LVSI), extensive intraductal component (EIC), estrogen receptor (ER) status, progesterone receptor status, human epidermal growth factor receptor 2, and omission of adjuvant radiation therapy. These factors can be grouped into three groups: patient factors, surgical factors, and tumor biology. Most studies do not distinguish between local IBTR and regional IBTR, mainly because they were comparing BCT with mastectomy, and any IBTR was considered a failure of BCT that mastectomy could have potentially prevented.

Young age is consistently reported to be an independent predictor of IBTR and, in general, younger patients are at higher risk. ${ }^{11,13-18}$ One hypothesis is that menopausal status and its interaction with breast cancer hormonal receptors rather than exact age more closely predict recurrence risk. The 15-year follow-up of the National Surgical Adjuvant

Table I Criteria for accelerated partial breast irradiation appropriateness by consensus statement or clinical trial

\begin{tabular}{|c|c|c|c|c|c|c|c|}
\hline & ASBS $^{6}$ & $\begin{array}{l}\text { ASTRO } \\
\text { "suitable"4 }\end{array}$ & $\begin{array}{l}\text { ASTRO } \\
\text { "cautionary"4 }\end{array}$ & $\begin{array}{l}\text { GEC-ESTRO } \\
\text { low-risk group }^{7}\end{array}$ & $\begin{array}{l}\text { GEC-ESTRO } \\
\text { intermediate- } \\
\text { risk group }^{7}\end{array}$ & $\begin{array}{l}\text { NSABP B-393 } \\
\text { (in progress) }\end{array}$ & $\mathrm{ABS}^{5}$ \\
\hline Published & 2008 & 2009 & 2009 & 2010 & 2010 & 2011 & 2013 \\
\hline \multicolumn{8}{|c|}{ Risk of local IBTR } \\
\hline Tumor size & $\leq 3 \mathrm{~cm}$ & $\leq 2 \mathrm{~cm}$ & $2.1-3.0 \mathrm{~cm}$ & $\leq 3 \mathrm{~cm}$ & $\leq 3 \mathrm{~cm}$ & $\leq 3 \mathrm{~cm}$ & $\leq 3 \mathrm{~cm}$ \\
\hline Margin & Negative & $\geq 2 \mathrm{~mm}$ & Close $(<2 \mathrm{~mm})$ & $\geq 2 \mathrm{~mm}$ & Close $(<2 \mathrm{~mm})$ & Negative & Negative \\
\hline $\begin{array}{l}\text { Hormonal } \\
\text { status }\end{array}$ & - & Positive & Negative & Any & Any & Any & Any \\
\hline \multicolumn{8}{|c|}{ Risk of regional IBTR } \\
\hline Histology & Any & Ductal & Lobular & Ductal & Any & Any & Any \\
\hline LVSI & - & None & Limited & None & None & Yes & None \\
\hline Pure DCIS & Yes & No & Yes & No & Yes & Yes & Yes \\
\hline Multifocal & - & $\begin{array}{l}\text { Clinically unifocal, } \\
\leq 2 \mathrm{~cm} \text { total }\end{array}$ & $\begin{array}{l}\text { Clinically unifocal, } \\
\leq 3 \mathrm{~cm} \text { total }\end{array}$ & Unifocal & $\begin{array}{l}\text { Yes, within }<2 \mathrm{~cm} \\
\text { of index lesion }\end{array}$ & $\begin{array}{l}\text { Microscopic, } \\
<3 \mathrm{~cm} \text { total }\end{array}$ & - \\
\hline Multicentric & - & Unicentric & Unicentric & Unicentric & Unicentric & Unicentric & - \\
\hline \multicolumn{8}{|c|}{ Risk of nodal or distant failure } \\
\hline Age (years) & $\begin{array}{l}\geq 45 \text { if invasive, } \\
\geq 50 \text { if DCIS }\end{array}$ & $\geq 60$ & $50-59$ & $\geq 50$ & $>40-50$ & $\geq 18$ & $\geq 50$ \\
\hline Nodal status & pNO & $\mathrm{pNO}$ & pNo & $\mathrm{pNO}$ & $\begin{array}{l}\mathrm{pNImi}, \mathrm{pN} \mathrm{la} \\
\text { (by ALND) }\end{array}$ & $\begin{array}{l}\text { pNO-I* } \\
\text { no ECE }\end{array}$ & $\mathrm{pNO}$ \\
\hline
\end{tabular}

Note: *At least six axillary nodes pathologically evaluated if $\mathrm{pNI}$.

Abbreviations: ABS, American Brachytherapy; ALND, axillary lymph node dissection; ASBS, American Society of Breast Surgeons; ASTRO, American Society for Therapeutic Radiology and Oncology; DCIS, ductal carcinoma in situ; ECE, extracapsular extension; GEC-ESTRO, Groupe Européen de Curiethérapie and European Society for Radiotherapy and Oncology; IBTR, ipsilateral breast tumor recurrence; LVSI, lymphovascular space invasion; NSABP, National Surgical Adjuvant Breast and Bowel Project; $\mathrm{pN}$, pathologic nodal status; -, not included in the criteria. 
Breast and Bowel Project (NSABP) B-06 randomized trial showed that both age less than 45 and over 65 years old predicted IBTR.$^{14}$ Wapnir et al evaluated over 2,600 patients enrolled in the NSABP B-17 and B-24 trials and showed a 2.1-fold increased risk of invasive IBTR in patients less than 45 years old compared with those aged 65 years and older. ${ }^{13}$ In regards to APBI, Ott et al evaluated a retrospective series of 274 patients treated with interstitial brachytherapy-based APBI and found that age less than 50 years old was associated with inferior local control. ${ }^{18}$

Histopathological examination of the lumpectomy specimen provides additional risk factors for IBTR including surgical margin status and tumor diameter. Patients with involved surgical margins were excluded from most prospective randomized trials and there is limited data informing the effect of APBI in the setting of positive margins. Recent expert consensus states that a positive surgical margin is associated with a twofold increase in the rate of IBTR. ${ }^{19}$ Large tumor size (by palpation or pathologic measurement) has a significant effect on local tumor control. ${ }^{15}$ Cefaro et al identified tumor size $\geq 3 \mathrm{~cm}$ was an independent significant factor predicting for local recurrence (a common criteria found among APBI consensus statements). ${ }^{20}$ Interestingly, tumor size has not been associated with IBTR following APBI. ${ }^{17}$ This result should be interpreted with caution because of the selection bias in patients considered eligible for APBI, which is typically limited to patients with tumors smaller than $3 \mathrm{~cm}^{4}$

As have been well described within the literature, factors indicative of tumor biology that have been independently associated with local recurrence include high grade, the presence of comedonecrosis, LVSI, and EIC. ${ }^{11-17,21,22}$ Also, there is increasing evidence that hormone receptor status plays a role in recurrence risk. In a pooled analysis of almost 2,000 patients, Shah et al found ER negativity to be the only independent predictor of local failure. ${ }^{23}$

\section{Factors predicting for regional IBTR: "Elsewhere Failures"}

Although critical to the success of APBI, there has been variability in the reporting of patterns of failure. Many series report out-of-field IBTRs as a local failure or as a new breast tumor primary. Although either may be true, it is our opinion that series of APBI should evaluate these recurrences as a distinct failure pattern, particularly when brachytherapy is used, in order to understand the pattern of recurrence relative to the conformal brachytherapy dose distribution. Regional IBTR describes tumor recurrence in a location that would be treated to full dose with WBI or excised with total mastectomy but receives negligible dose with APBI. The distinction between a non-contiguous recurrence versus a new ipsilateral primary is of little consequence in evaluating the safety of APBI because any in-breast disease would have been presumably treated with WBI or mastectomy. Recht et al defined this entity as an "elsewhere failure": recurrent disease arising within the ipsilateral breast but at least a few centimeters from the lumpectomy cavity. ${ }^{24}$ Recent pooled series would predict regional IBTR to be on the order of $2 \%$ at 5 years for patients eligible for and receiving APBI. ${ }^{23}$

Considering the variability of reporting within the published series, it is currently difficult to provide factors that would predispose to regional IBTR as opposed to local IBTR. Despite this, there are several factors that are likely to contribute. Multicentric breast cancer is, by definition, regional breast disease that is unlikely to be treated with most APBI techniques except under unique circumstances. Similarly, multifocal breast cancer is likely a risk factor for regional IBTR, conceivably because a negative surgical margin could be reported by bisecting breast tissue separating two distinct islands of tumor cells and therefore leaving disease in the breast. This is reflected in the American Society for Therapeutic Radiology and Oncology (ASTRO) consensus guidelines by permitting microscopic multifocality less than $3 \mathrm{~cm}$ total, but not clinically apparent multifocality or islands of tumor spanning more than $3 \mathrm{~cm}^{4}$

An EIC not only increases the total tumor diameter but has been shown to predict for residual disease after lumpectomy in older mastectomy series. ${ }^{25}$ Modern reports, however, demonstrate that EIC excised to negative margin is not a risk factor for local recurrence after lumpectomy and WBI. ${ }^{26}$ Despite this, when treated with interstitial and balloon brachytherapy-based APBI, local failure was higher in patients with EIC. ${ }^{27,28}$ As a result, there are mixed recommendations regarding APBI in patients with EIC and negative surgical margins.

Similar to EIC, APBI for pure ductal carcinoma in situ (DCIS) is somewhat controversial. This is largely based on the fact that the majority of currently available prospective trials of APBI have excluded pure DCIS. However, recent pooled retrospective data show excellent local control after APBI with DCIS. ${ }^{16,29,30}$

The multicentric natural history of invasive lobular carcinoma (ILC) led some experts to question the use of APBI in these patients, and there is limited retrospective evidence and no data from randomized trials to evaluate ILC's significance to IBTR, likely because this histology was excluded from 
many of the historic BCT studies. Additionally, some of the first clinical results of APBI supported this concern. ${ }^{10,31}$ Since these study protocols have been developed, however, several modern series clinical data have demonstrated that APBI with brachytherapy is safe in patients with ILC, ${ }^{17,18,32}$ and consensus groups have revised their criteria to include lobular histology. ${ }^{5}$ Notably, any lobular histology remains "cautionary" under the current ASTRO consensus. ${ }^{4}$

Although LVSI is a prognostic factor in breast cancer, its significance in APBI patient selection is disputed because of conflicting existing evidence. A single institution reported on 106 patients defined as "cautionary" under ASTRO grouping who underwent APBI. ${ }^{33}$ Among three recurrences, focal LVSI was not predictive of failure. To the contrary, a similar study including 277 patients who received brachytherapybased APBI reported that LVSI was indeed predictive of local failure. ${ }^{27}$ Although focal LVSI is permitted by ASTRO, any presence of LVSI is considered as "unsuitable" under American Brachytherapy Society (ABS) consensus ${ }^{5}$ mainly based on a lack of existing data.

\section{Factors predicting for nodal or distant failure}

There is little data evaluating risk factors for nodal or distant disease recurrence in patients treated with APBI because most providers would not recommend APBI in patients who are at substantial risk for such a failure. This patient selection bias results in a low incidence of nodal and distant failure and conclusions are difficult to draw, but several important aspects should be highlighted.

The use of APBI in patients with node-positive breast cancer is controversial and in general should not be done outside a clinical trial. Patients with pN1 without extracapsular extension were eligible for enrollment on NSABP B-39 if at least six axillary lymph nodes were examined. ${ }^{3}$ However, there are some small series demonstrating that it may be an appropriate technique in carefully selected patients. ${ }^{34}$ In order to select patients at sufficiently low risk of recurrence outside the breast several factors are considered. Perhaps most important is the surgical evaluation of lymphatic basins. Sentinel lymph-node biopsy (SLNB) generally carries a 7.3\% false negative rate but this rate can vary widely $(0 \%-29 \%) .{ }^{35}$ The significance of a false negative rate of SLNB is mitigated somewhat for patients receiving WBI, due to the fact that standard WBI with opposed tangent photon beams typically includes most of axillary level I lymph nodes. In APBI, however, dose to the axilla is negligible. Under these assumptions, patients receiving APBI are at a real risk for a node-only failure. This reasoning underscores the importance of patient selection and appropriate utilization of adjuvant systemic therapy. During patient selection, factors such as lymph-node biopsy technique as well as primary tumor location, size, and grade should be carefully considered to ensure an adequately high negative predictive value (though a low pretest probability).

Receptor status has become a matter of paramount importance in breast cancer prognosis and treatment. Patients with ER-positive breast cancer have lower rates of nodal and distant failure in general, and this finding has been demonstrated in patients treated with APBI as well. ${ }^{23,36}$ Although local control remains excellent, patients with triple negative disease have demonstrated a higher rate and earlier onset of nodal and distant recurrence. ${ }^{37}$ Consequently, most consensus guidelines include receptor status in APBI eligibility criteria.

\section{Consensus group recommendations}

Several cooperative groups including ASTRO, the ABS, American Society of Breast Surgeons (ASBS), and the Groupe Européen de Curiethérapie and European Society for Radiotherapy and Oncology (GEC-ESTRO) have published consensus guidelines on the appropriate selection of patients for APBI. ${ }^{3-6}$ These recommendations are based on clinical evidence from randomized trials, prospective studies, and expert opinion. The selection criteria recommended by each of these groups (ASTRO, ABS, ASBS, and GEC-ESTRO) are outlined in Table 1. Additionally, provided are the inclusion criteria for the current ongoing clinical trial, NSABP B-39, which is evaluating the use of APBI in comparison with standard WBI. ${ }^{3}$

As demonstrated, the consensus group recommendations align regarding many APBI criteria including size $(<3 \mathrm{~cm})$ and margin status (negative or $<2 \mathrm{~mm}$ ). However, there is some variability in the recommendations regarding histology, the presence of DCIS, EIC, and multifocal tumors. It is well documented that older age is linked to higher local control and all current guidelines agree that patients $\geq 50$ years old should not be excluded from APBI based on age alone. NSABP B-39 has included all patients $\geq 18$ years old, which may provide further clarification regarding which patients should be eligible for APBI.

As discussed earlier, there is limited evidence regarding APBI in patients with ILC, and consensus recommendations are mixed. While it is excluded from the "suitable" and "lowrisk" groups of the ASTRO and GEC-ESTRO consensus guidelines, it is not included in the final consideration of the other consensus statements. 
EIC was associated with a higher risk of IBTR in the MammoSite registry data, thus patients with EIC $\leq 3 \mathrm{~cm}$ are considered as ASTRO "cautionary" and those with EIC $>3 \mathrm{~cm}$ are "unsuitable". Similarly, pure DCIS $\leq 3 \mathrm{~cm}$ is considered as "cautionary" while more extensive DCIS is "unsuitable".

Regarding multifocal disease, the GEC-ESTRO guideline excludes multifocal tumors from the low-risk group, given the lack of published data on outcomes with these patients. ASBS and ABS do not comment on multifocality and the NSABP B-39 inclusion criteria has followed ASTRO allowing microscopic multifocality $<3 \mathrm{~cm}$ total.

Candidates for APBI should undergo axillary staging with either SLNB or axillary node dissection. Data from the early APBI trials that did not have complete pathologic axillary staging suggest higher rates of recurrence in those patients treated with APBI. ${ }^{31}$ All consensus guidelines (except for the GEC-ESTRO “intermediate-risk” group) advise against the use of APBI in node-positive patients. Patients should have documented node negative or only isolated foci $(<0.2 \mathrm{~mm})$ of microscopic tumor cells to be considered as "suitable", as per ASTRO recommendations. A recent and useful nomogram developed by Wobb et al provides a method for predicting locoregional failure based on several of the factors discussed here. ${ }^{36}$

\section{Potential criticisms of consensus guidelines}

Analysis of patients treated with APBI in recent years has identified some limitations in the current consensus statement guidelines. To date, only a single study has shown a statistically significant difference in IBTR between ASTRO consensus statement groups following APBI ${ }^{38}$ Other studies have demonstrated that the current ASTRO consensus guidelines are not predictive of IBTR in patients treated with APBI, ${ }^{39,40}$ and that "cautionary" group patients have acceptably low rates of IBTR. ${ }^{33,41-43}$ Although the follow-up duration of many of these series remains less than 10 years, they will undoubtedly contribute to refinement of the consensus guidelines as they continue to mature.

A recent pooled analysis of a large cohort of patients treated at William Beaumont Hospital and the ASBS MammoSite Registry reports no significant difference in the rate of IBTR or true recurrence/marginal miss based on ASTRO consensus statement groups. ${ }^{39}$ Patients in the "cautionary" and "unsuitable" categories did have trend toward higher rates of regional IBTR and new primaries. The authors of this large cohort analysis propose that the current ASTRO guidelines fail to adequately differentiate patients at increased risk of regional IBTR from local IBTR, likely due to the limited available clinical data on APBI.

As aforementioned, data on the outcomes of pure DCIS treated with APBI are limited. Pure DCIS is currently considered as "cautionary" on the ASTRO consensus guidelines and "intermediate risk" on the GEC-ESTRO guidelines. There is some evidence supporting APBI in patients with pure DCIS. A study of over 1,600 women treated with APBI has shown no association between DCIS and risk of local recurrence. ${ }^{17}$ Additionally, a recent analysis by Vicini et al reports excellent outcomes of 300 women with DCIS treated with APBI with no significant difference in the rate of IBTR between the cautionary DCIS group compared with both a pooled suitable/cautionary invasive group and invasive "suitable" risk patients. ${ }^{29}$ These data along with several other series with similar results suggest that patients with pure DCIS less than $3 \mathrm{~cm}$ total could be included in the "suitable" category and safely treated with APBI. ${ }^{16,33,41}$

While there is likely overlap between the factors predicting local IBRT and those predicting regional IBTR, some patients may fail regardless of treatment modality and may have been eligible for the BCT with WBI. McHaffie et al demonstrated that women with DCIS or age 50-59 as their only "cautionary" risk factor that otherwise would have met "suitable" criteria had excellent locoregional control compared with other "cautionary" patient factors. ${ }^{41}$ Several other recent analyses of patients treated with APBI have shown that ER-negative tumors have a higher local recurrence compared with ER-positive patients. ${ }^{33,43}$ Beitsch et al identified ER-negative status as the only predictor of 5-year IBTR following APBI. ${ }^{42}$ Together, the presented studies provide evidence for revisions in the current consensus statements that are likely to occur as data mature.

\section{Brachytherapy-specific considerations High dose rate versus low dose rate}

Low dose-rate (LDR) brachytherapy isotopes have been the gold standard for brachytherapy sources for decades, including in breast APBI. ${ }^{44,45}$ In recent years, however, there has been a global increase in the utilization of high dose-rate (HDR) sources. The reasons for this are multifactorial but include improved patient and staff safety, convenience, and potentially a radiobiological advantage. HDR first demonstrated effectiveness compared with LDR when delivered as a local boost after WBI. ${ }^{46,47}$ There were concerns, however, that an increase in dose rate could have radiobiological effects that could result in inferior cosmesis or possibly tumor control. 
The Radiation Therapy Oncology Group (RTOG) compared LDR sources with HDR sources for APBI in a phase II trial accruing in the late 1990s (RTOG 9517). LDR prescription was 45 Gy over 3.5-5 days and HDR was 34 Gy in 10 fractions twice-daily over 5 days. Early results demonstrated less grade 3-4 toxicity within the HDR arm, ${ }^{48}$ and with 6-year follow-up there were similar rates and patterns of failure between arms. ${ }^{49}$ Considering the advantages in safety and convenience with HDR brachytherapy, HDR sources like Iridium-192 have replaced LDR for the treatment of breast cancers in many radiation therapy centers in the United States.

The Xoft Axxent electronic brachytherapy device (Xoft, Inc., Sunnyvale, CA, USA) is a more recent development in brachytherapy that has demonstrated usefulness in APBI since its Food and Drug Administration (FDA) approval in 2006. ${ }^{50-52}$ This device utilizes electricity to create a low energy $(50 \mathrm{kV})$ source similar to that of other APBI techniques. ${ }^{53,54}$ An obvious benefit of the Xoft system is that it is not radioactive, which could further improve patient and staff safety. Additionally, these low energy sources do not require the intensive shielding needed for iridium sources and therefore can be used in unshielded operating rooms. The drawbacks to this low energy source include the limiting surface dose (at the surface of the applicator), a problem that can be overcome with HDR sources, which can have a more homogeneous dose distribution. ${ }^{54,55}$

\section{Applicator selection}

There are numerous applicators available for use in breast brachytherapy and applicator selection should depend on patient-specific anatomy and surgical findings, but selection is commonly limited by applicator availability and user expertise. The first breast brachytherapy applicators reported were breast bridges, in which interstitial needles were placed through a template into the breast. ${ }^{46,47,49}$ This technique has the advantage of being highly customizable although user technique is critical and treatment planning is time intensive. The Harrison-Anderson-Mick silastic applicator (Mick Radio-Nuclear instruments, Mount Vernon, NY, USA) can be thought of as several needles fixed within a rectangular silicone mold. Although the Harrison-AndersonMick applicator has demonstrated clinical effectiveness, ${ }^{56,57}$ depending on the shape of the lumpectomy cavity it can be bulky. Revisions to this design have been made but they remain in early stages of development. ${ }^{56}$

The most popular applicator devices in the US are balloon-based. These applicators are placed directly into the lumpectomy cavity and inflated with water or saline (with a small amount of contrast material added). This process provides a customized treatment plan for each patient and improves dose homogeneity. Original balloon-based applicators like the MammoSite single-lumen (Hologic, Marlborough, MA, USA) contained a single channel for the HDR source. Although dose could be optimized longitudinally (along the path of the source), radial dose was always uniform (perpendicular to the path of the source). The addition of multiple catheters through the balloon has allowed for radial dose variation and optimization, and has made balloon-based brachytherapy more customizable..$^{55,58,59}$

Several additional lumpectomy HDR applicators that serve as a hybrid of those listed previously are available and early clinical results are favorable. These include the strut adjusted volume implant ${ }^{60-63}$ (Cianna Medical, Aliso, Viejo, CA, USA) and the ClearPath ${ }^{64}$ (North American Scientific, Chatsworth, CA, USA). A comprehensive review summarizing the pros and cons of various APBI applicators is outside the scope of this review but available within the existing literature. ${ }^{65}$

\section{Noninvasive image-guided breast brachytherapy}

Brachytherapy has historically referred to the placement of radioactive isotopes into or adjacent to the tumor bed, but the distinction between this "traditional" brachytherapy and external beam RT has become increasingly blurred with advancements in technology. An example illustrating this point is noninvasive image-guided breast brachytherapy (NIBB). To the authors' knowledge, AccuBoost (Advanced Radiation Therapy, Inc., Billerica, MA, USA) is the only FDA-approved device for NIBB to date. This device involves breast immobilization with compression and $\mathrm{kV}$ orthogonal imaging. After planning and optimization, an HDR afterloader delivers the prescription dose from opposed orthogonal beams to the tumor bed (four total beams). Although most widely used as a tumor bed boost given prior to $\mathrm{WBI},{ }^{66-68}$ early results suggest that NIBB can be used safely for APBI. ${ }^{69}$ Mature data demonstrating the efficacy for NIBB for APBI, and compared with other APBI techniques, are pending.

\section{Image-guided brachytherapy}

Although early breast brachytherapy (specifically intraoperative RT [IORT]) was delivered without image guidance, the use of imaging modalities used to confirm the proper placement of the applicator and improve dose optimization has become a critical component of brachytherapy. Image guidance can also serve to abort applicator placement in patients thought to not be safe for breast brachytherapy, 
such as having too high of a projected skin dose or too large of a lumpectomy cavity. One study reported that this frequency could be as high as $19 \%$ of patients planned for IORT. ${ }^{70}$

The images collected with the applicator in situ can provide a means of optimizing the dose delivered and sparing adjacent normal structures. First reported for APBI in 1997, ultrasound has now become the most ubiquitous modality used for postoperative applicator placement. ${ }^{71-73}$ Benefits to ultrasound include that it is easy to use, inexpensive, and non-ionizing. If the applicator is placed in the operating room, then ultrasound can also be used to confirm applicator positioning and the lack of air within the lumpectomy cavity. ${ }^{53}$

The sensitivity of mammography in detecting breast abnormalities makes it ideal for use as image guidance in brachytherapy. Although portal and $\mathrm{kV}$ imaging has been used in breast brachytherapy since its inception, high-quality mammography has been used minimally for guidance until recent years. The AccuBoost system utilizes its on-board $\mathrm{kV}$ imaging system to collect orthogonal compression mammograms for lumpectomy cavity localization and planning. Although early toxicity results are favorable, clinical outcomes are currently pending. ${ }^{66-69}$

Computed tomography (CT) scanners are common within RT centers and RT staff is well accustomed to the CT-plan-treat workflow. In the case of needle-based brachytherapy applicators, CT guidance may provide benefit in needle placement and resultant dosimetry. ${ }^{74-76}$ For balloonbased applicators, CT imaging can also provide aid in terms of placement and improved computer-based planning and optimization. ${ }^{55,77}$

Although the role of magnetic resonance (MR) imaging in breast brachytherapy remains to be defined, there are several potentially interesting applications. Jolicoeur et al reported on 70 patients who underwent CT- and MR-based simulation and APBI planning at their institution. ${ }^{78}$ Their results suggest that MR-based planning results in a smaller and more accurately defined target volume than CT-based planning. They comment that MR-based simulation could obviate the need for surgical clip placement at the time of lumpectomy. The use of CT+MR imaging in breast brachytherapy will provide for interesting analyses in the future.

\section{Intraoperative brachytherapy}

IORT is a form of APBI that has been reported in two large European trials with favorable outcomes using $\mathrm{kV}$ photon ${ }^{79}$ and $\mathrm{MV}$ electron ${ }^{80}$ techniques. Although a full comparative discussion is outside the scope of this review, there are several logistic and dosimetric concerns to external beam-based
IORT, and breast brachytherapy is poised to overcome several of these drawbacks. ${ }^{81}$

Although still in an investigational phase, balloonbased HDR breast IORT can be utilized to give a focused, homogeneous dose to a small volume of breast tissue. ${ }^{55} \mathrm{At}$ our institution, we have an active protocol accruing patients to receive an IORT single 12.5 Gy dose prescribed to $1 \mathrm{~cm}$ from the balloon surface, which provides a dose chosen to deliver a similar surface dose as the $\mathrm{kV}$ photon IORT trial. A balloon-based multichannel catheter is placed immediately following lumpectomy and a planning CT scan is performed using in-room CT imaging. RT is delivered using an HDR source (Iridium-192) to $1 \mathrm{~cm}$ from the surface of the balloon. ${ }^{55}$ Although the procedure is performed in a dedicated brachytherapy suite with full anesthesia capabilities, HDR shielding, and a CT-on-rails system, ${ }^{82}$ we believe that the results will provide meaningful insight into breast IORT that can be expanded to centers with more standard HDR delivery systems.

\section{Dose and fractionation}

Akin to the debate over the optimal external beam dose and fractionation, considerable debate exists over the optimal dose and fractionation of HDR breast brachytherapy that optimizes tumor control and minimizes toxicity. The optimal dose and fractionation for use in APBI with brachytherapy is a subject for debate. Breast brachytherapy was first validated with LDR doses of 50 Gy given over 4-5 days following inpatient admission. ${ }^{44,83}$ Since then, LDR doses have ranged from 20 to 60 Gy in select series. ${ }^{3,84}$ As discussed earlier, however, LDR utilization is being replaced at many centers with HDR sources.

The earliest and largest series of APBI with HDR brachytherapy deliver 34-38 Gy twice-daily separated by 6 hours over 5 days (10 total fractions). ${ }^{3,49,85,86}$ However, the "typical dose" of 34 Gy over 10 fractions is hardly the only dose validated and a wide range of HDR doses have been reported from 20 Gy in 2 fractions to 37.2 Gy in 10 fractions. ${ }^{3,85}$

The utilization of IORT will provide an interesting extension into the breast cancer alpha/beta ratio and, consequently, brachytherapy dosing and fractionation. If single fraction IORT provides equivalent long-term local control and toxicity outcomes, there will likely be a movement toward single fraction postoperative APBI using breast brachytherapy. A caution, of course, is that many forms of breast IORT utilize unique delivery mechanisms (eg, $\mathrm{kV}$ photons) that are not directly biologically comparable to HDR balloon-based brachytherapy. To date, there have been no randomized trials 
directly comparing HDR doses used in breast brachytherapy and the majority of reported data (as well as the current national cooperative group trial) ${ }^{3}$ recommend 34 Gy in 10 fractions twice-daily as the standard APBI dose..$^{3,28}$

\section{Post-augmentation or reconstruction}

APBI is particularly difficult when cancer is detected in a breast following augmentation, when the placement of the capsule can distort normal breast anatomy and risk contracture following RT. Brachytherapy in an augmented breast has been described for over 20 years, ${ }^{87,88}$ however, only case reports have been published using strut-adjusted volume implants ${ }^{89}$ or multilumen balloon applicators in the augmented breast. ${ }^{90}$ These case reports each demonstrate favorable dosimetric and cosmetic results, although larger series are yet unpublished.

Distortion of the tumor bed at the time of lumpectomy through autologous tissue transfer (ie, oncoplastic reconstruction) results in difficulty defining the target volume for APBI. Recently, Roth et al retrospectively analyzed 134 patients who received oncoplastic surgery followed by interstitial needlebased ABPI. ${ }^{91}$ They utilized pathologic evaluation, physical exam, and all available imaging to define a customized target volume for each patient. Their results suggest that breast brachytherapy is safe following oncoplastic surgery as there were few recurrences, but the median follow-up duration was 39 months and more mature data are pending.

\section{Implanted cardiac devices}

Photon interactions with implanted cardiac devices (ie, pacemakers and defibrillators) can result in a life-threatening malfunction depending on the patient's cardiac disease and device status. Increasing the distance between the radioactive source and the device provides the most reduction in dose delivered to it. Unfortunately, unless the cardiac device is relocated to the other side of the chest, the distance between the lumpectomy cavity and the device is relatively fixed. Depending on patient anatomy, breast brachytherapy would likely offer a reduction in dose delivered to the device compared with standard WBI and external beam APBI. Although limited and highly patient specific, there are several small series of balloon-based breast brachytherapy in patients with cardiac devices demonstrating favorable dosimetric and clinical outcomes. ${ }^{92-94}$

\section{Brachytherapy for local recurrence}

Given the favorable survival outcomes demonstrated in women with early stage breast cancer treated with BCT, an increasing number of women may present with breast cancer in an intact, previously irradiated breast. Historically, mastectomy had been the standard of care for these IBTR (or ipsilateral new primary) tumors. Currently, the RTOG 1,014 trial is evaluating the use of repeat breast conservation and 3-D conformal re-irradiation in women previously treated with lumpectomy and whole breast radiation. ${ }^{95}$ There are currently no randomized controlled trials to evaluate the use of breast conservation with brachytherapy for locally recurrent disease; however, various single-institution studies have reported their experience.

There are at least three separate single-institution reports of brachytherapy after second lumpectomy for recurrent breast cancer after WBI. Together, the mastectomy-free survival ranges from $73 \%$ to $94.4 \%$ with limited follow-up to date. ${ }^{96-98}$ In a phase II study by Chadha et al, 15 patients with a localized IBTR underwent a second lumpectomy and LDR brachytherapy to 30 or 45 Gy. ${ }^{96}$ They reported excellent overall survival and local control (ie, mastectomy free survival) rates of $100 \%$ and $89 \%$, respectively, with a median of 36 months follow-up. The single patient with a local recurrence at 27 months received 30 Gy. Further, there was no grade 3 or 4 toxicity reported. These data suggest that patients previously treated with breast conservation may be candidates for re-irradiation with acceptable toxicity and outcomes, although further prospective data are warranted.

\section{Conclusion}

APBI is aimed to prevent failure within the lumpectomy cavity but not necessarily elsewhere. As such, selecting patients at high enough risk for local failure to warrant adjuvant therapy but a low enough risk for out of field failures (regional IBTR, regional nodal recurrence, and distant metastasis) is a key to select patients who will benefit from APBI. Brachytherapy can provide focused, high-dose RT to the area at highest risk for local failure and is an excellent option. APBI needs to be customized to each patient based on anatomy, patient comorbidities, and tumor-specific characteristics and these are the each hallmark of brachytherapy. APBI brachytherapy can be considered in select cases and these patients should be referred to specialized centers with sufficient expertise. Results of several large international trials are pending, which will guide further recommendations regarding APBI and breast brachytherapy. Until then several guidelines exist for patient selection, and a careful examination of the rationale behind these recommendations provides meaningful insight into the disease biology. 


\section{Disclosure}

The authors report no conflicts of interest in this work.

\section{References}

1. National Comprehensive Cancer Network Clinical Practice Guidelines: Breast Cancer v 2.2015 [database on the Internet]. Available from: http:// www.nccn.org/professionals/physician_gls/pdf/breast.pdf. Accessed April 10, 2015.

2. Fisher B, Anderson S, Bryant J, et al. Twenty-year follow-up of a randomized trial comparing total mastectomy, lumpectomy, and lumpectomy plus irradiation for the treatment of invasive breast cancer. N Engl J Med. 2002;347(16):1233-1241.

3. Wolmark N, Curan WJ, Vicini F, et al. NASBP Protocol B-39 (RTOG Protocol 0413): a randomized phase III study of conventional whole breast irradiation (WBI) versus partial breast irradiation (PBI) for women with stage 0 , I, or II breast cancer [database on the Internet] 2011. Available from: http://rpc.mdanderson.org/rpc/credentialing/files/ B39_Protocol1.pdf.

4. Smith BD, Arthur DW, Buchholz TA, et al. Accelerated partial breast irradiation consensus statement from the American Society for Radiation Oncology (ASTRO). Int J Radiat Oncol Biol Phys. 2009; 74(4):987-1001.

5. Shah C, Vicini F, Wazer DE, Arthur D, Patel RR. The American Brachytherapy Society consensus statement for accelerated partial breast irradiation. Brachytherapy. 2013;12(4):267-277.

6. The American Society of Breast Surgeons: Consensus Statement for Accelerated Partial Breast Irradiation [database on the Internet]. 2008. Available from: https://www.breastsurgeons.org/statements/ PDF_Statements/APBI_statement_revised_100708.pdf. Accessed April 10, 2015.

7. Polgar C, Van Limbergen E, Potter R, et al. Patient selection for accelerated partial-breast irradiation (APBI) after breast-conserving surgery: recommendations of the Groupe Europeen de Curietherapie-European Society for Therapeutic Radiology and Oncology (GEC-ESTRO) breast cancer working group based on clinical evidence (2009). Radiother Oncol. 2010;94(3):264-273.

8. Mould RF. Invited review: the early years of radiotherapy with emphasis on X-ray and radium apparatus. Br J Radiol. 1995;68(810):567-582.

9. Husain ZA, Mahmood U, Hanlon A, et al. Accelerated partial breast irradiation via brachytherapy: a patterns-of-care analysis with ASTRO consensus statement groupings. Brachytherapy. 2011;10(6): 479-485.

10. Kurtz JM, Amalric R, Brandone H, Ayme Y, Spitalier JM. Results of wide excision for mammary recurrence after breast-conserving therapy. Cancer. 1988;61(10):1969-1972.

11. Bijker N, Peterse JL, Duchateau L, et al. Risk factors for recurrence and metastasis after breast-conserving therapy for ductal carcinomain-situ: analysis of European Organization for Research and Treatment of Cancer Trial 10853. J Clin Oncol. 2001;19(8):2263-2271.

12. Fisher B, Anderson S, Redmond CK, Wolmark N, Wickerham DL, Cronin WM. Reanalysis and results after 12 years of follow-up in a randomized clinical trial comparing total mastectomy with lumpectomy with or without irradiation in the treatment of breast cancer. $N$ Engl $J$ Med. 1995;333(22):1456-1461.

13. Wapnir IL, Dignam JJ, Fisher B, et al. Long-term outcomes of invasive ipsilateral breast tumor recurrences after lumpectomy in NSABP B-17 and B-24 randomized clinical trials for DCIS. J Natl Cancer Inst. 2011;103(6):478-488.

14. Fisher ER, Anderson S, Tan-Chiu E, Fisher B, Eaton L, Wolmark N. Fifteen-year prognostic discriminants for invasive breast carcinoma: National Surgical Adjuvant Breast and Bowel Project Protocol-06. Cancer. 2001;91(8 Suppl):1679-1687.

15. Bartelink H, Horiot JC, Poortmans P, et al. Recurrence rates after treatment of breast cancer with standard radiotherapy with or without additional radiation. $N$ Engl J Med. 2001;345(19):1378-1387.
16. Jeruss JS, Kuerer HM, Beitsch PD, Vicini FA, Keisch M. Update on DCIS outcomes from the American Society of Breast Surgeons accelerated partial breast irradiation registry trial. Ann Surg Oncol. 2011; 18(1):65-71.

17. Shah C, Wilkinson JB, Shaitelman S, et al. Clinical outcomes using accelerated partial breast irradiation in patients with invasive lobular carcinoma. Int J Radiat Oncol Biol Phys. 2011;81(4):e547-e551.

18. Ott OJ, Hildebrandt G, Potter R, et al. Accelerated partial breast irradiation with interstitial implants: risk factors associated with increased local recurrence. Int J Radiat Oncol Biol Phys. 2011;80(5):1458-1463.

19. Moran MS, Schnitt SJ, Giuliano AE, et al. Society of Surgical OncologyAmerican Society for Radiation Oncology consensus guideline on margins for breast-conserving surgery with whole-breast irradiation in stages I and II invasive breast cancer. J Clin Oncol. 2014;32(14): $1507-1515$.

20. Cefaro GA, Genovesi D, Marchese R, et al. Predictors of local recurrence after conservative surgery and whole-breast irradiation. Breast Cancer Res Treat. 2006;98(3):329-335.

21. Borger J, Kemperman H, Hart A, Peterse H, van Dongen J, Bartelink H. Risk factors in breast-conservation therapy. J Clin Oncol. 1994; 12(4):653-660.

22. Connolly JL, Schnitt SJ. Evaluation of breast biopsy specimens in patients considered for treatment by conservative surgery and radiation therapy for early breast cancer. Pathol Annu. 1988;23 Pt 1:1-23.

23. Shah C, Wilkinson JB, Lyden M, Beitsch P, Vicini FA. Predictors of local recurrence following accelerated partial breast irradiation: a pooled analysis. Int J Radiat Oncol Biol Phys. 2012;82(5):e825-e830.

24. Recht A, Silver B, Schnitt S, Connolly J, Hellman S, Harris JR. Breast relapse following primary radiation therapy for early breast cancer. I Classification, frequency and salvage. Int J Radiat Oncol Biol Phys. 1985;11(7):1271-1276.

25. Holland R, Connolly JL, Gelman R, et al. The presence of an extensive intraductal component following a limited excision correlates with prominent residual disease in the remainder of the breast. J Clin Oncol. 1990;8(1):113-118.

26. Leong C, Boyages J, Jayasinghe UW, et al. Effect of margins on ipsilateral breast tumor recurrence after breast conservation therapy for lymph node-negative breast carcinoma. Cancer. 2004;100(9):1823-1832.

27. Cannon DM, McHaffie DR, Patel RR, et al. Locoregional recurrence following accelerated partial breast irradiation for early-stage invasive breast cancer: significance of estrogen receptor status and other pathological variables. Ann Surg Oncol. 2013;20(11):3446-3452.

28. Beitsch PD, Wilkinson JB, Vicini FA, et al. Tumor bed control with balloon-based accelerated partial breast irradiation: incidence of true recurrences versus elsewhere failures in the American Society of Breast Surgery MammoSite $\left({ }^{\circledR}\right)$ Registry Trial. Ann Surg Oncol. 2012;19(10): 3165-3170.

29. Vicini F, Shah C, Ben Wilkinson J, Keisch M, Beitsch P, Lyden M. Should ductal carcinoma-in-situ (DCIS) be removed from the ASTRO consensus panel cautionary group for off-protocol use of accelerated partial breast irradiation (APBI)? A pooled analysis of outcomes for 300 patients with DCIS treated with APBI. Ann Surg Oncol. 2013;20(4):1275-1281.

30. Shah C, Badiyan S, Ben Wilkinson J, et al. Treatment efficacy with accelerated partial breast irradiation (APBI): final analysis of the American Society of Breast Surgeons MammoSite $\left({ }^{\circledR}\right)$ breast brachytherapy registry trial. Ann Surg Oncol. 2013;20(10):3279-3285.

31. Ribeiro GG, Magee B, Swindell R, Harris M, Banerjee SS. The Christie Hospital breast conservation trial: an update at 8 years from inception. Clin Oncol (R Coll Radiol). 1993;5(5):278-283.

32. Strnad V, Hildebrandt G, Potter R, et al. Accelerated partial breast irradiation: 5-year results of the German-Austrian multicenter phase II trial using interstitial multicatheter brachytherapy alone after breastconserving surgery. Int J Radiat Oncol Biol Phys. 2011;80(1):17-24.

33. Stull TS, Catherine Goodwin M, Gracely EJ, et al. A single-institution review of accelerated partial breast irradiation in patients considered "cautionary" by the American Society for Radiation Oncology. Ann Surg Oncol. 2012;19(2):553-559. 
34. Shah C, Wilkinson JB, Shaitelman S, et al. Impact of lymph node status on clinical outcomes after accelerated partial breast irradiation. Int $J$ Radiat Oncol Biol Phys. 2012;82(3):e409-e414.

35. Kim T, Giuliano AE, Lyman GH. Lymphatic mapping and sentinel lymph node biopsy in early-stage breast carcinoma: a metaanalysis. Cancer. 2006;106(1):4-16.

36. Wobb JL, Chen PY, Shah C, et al. Nomogram for predicting the risk of locoregional recurrence in patients treated with accelerated partialbreast irradiation. Int J Radiat Oncol Biol Phys. 2015;91(2):312-318.

37. Wilder RB, Curcio LD, Khanijou RK, et al. Results with accelerated partial breast irradiation in terms of estrogen receptor, progesterone receptor, and human growth factor receptor 2 status. Int J Radiat Oncol Biol Phys. 2010;78(3):799-803.

38. Leonardi MC, Maisonneuve P, Mastropasqua MG, et al. How do the ASTRO consensus statement guidelines for the application of accelerated partial breast irradiation fit intraoperative radiotherapy? A retrospective analysis of patients treated at the European Institute of Oncology. Int J Radiat Oncol Biol Phys. 2012;83(3):806-813.

39. Wilkinson JB, Beitsch PD, Shah C, et al. Evaluation of current consensus statement recommendations for accelerated partial breast irradiation: a pooled analysis of William Beaumont Hospital and American Society of Breast Surgeon MammoSite Registry Trial Data. Int J Radiat Oncol Biol Phys. 2013;85(5):1179-1185.

40. Shaitelman SF, Vicini FA, Beitsch P, Haffty B, Keisch M, Lyden M. Five-year outcome of patients classified using the American Society for Radiation Oncology consensus statement guidelines for the application of accelerated partial breast irradiation: an analysis of patients treated on the American Society of Breast Surgeons MammoSite Registry Trial. Cancer. 2010;116(20):4677-4685.

41. McHaffie DR, Patel RR, Adkison JB, Das RK, Geye HM, Cannon GM. Outcomes after accelerated partial breast irradiation in patients with ASTRO consensus statement cautionary features. Int J Radiat Oncol Biol Phys. 2011;81(1):46-51.

42. Beitsch P, Vicini F, Keisch M, Haffty B, Shaitelman S, Lyden M. Five-year outcome of patients classified in the "unsuitable" category using the American Society of Therapeutic Radiology and Oncology (ASTRO) Consensus Panel guidelines for the application of accelerated partial breast irradiation: an analysis of patients treated on the American Society of Breast Surgeons MammoSite ${ }^{\circledR}$ Registry trial. Ann Surg Oncol. 2010;17(Suppl 3):219-225.

43. Christoudias MK, Collett AE, Stull TS, Gracely EJ, Frazier TG, Barrio AV. Are the American Society for Radiation Oncology guidelines accurate predictors of recurrence in early stage breast cancer patients treated with balloon-based brachytherapy? Int J Surg Oncol. 2013;2013:829050.

44. Vicini FA, Chen PY, Fraile M, et al. Low-dose-rate brachytherapy as the sole radiation modality in the management of patients with early-stage breast cancer treated with breast-conserving therapy: preliminary results of a pilot trial. Int J Radiat Oncol Biol Phys. 1997;38(2):301-310.

45. Seitz W, Stanek C, Stadler B, Binder W, Jakesz R, Reiner G. [Interstitial radiotherapy of breast cancer. Preliminary report of a prospective study of 165 cases subjected to breast-conserving treatment]. Strahlenther Onkol. 1990;166(10):654-658.

46. Resch A, Potter R, Van Limbergen E, et al. Long-term results (10 years) of intensive breast conserving therapy including a high-dose and largevolume interstitial brachytherapy boost (LDR/HDR) for T1/T2 breast cancer. Radiother Oncol. 2002;63(1):47-58.

47. Chen PY, Vicini FA, Benitez P, et al. Long-term cosmetic results and toxicity after accelerated partial-breast irradiation: a method of radiation delivery by interstitial brachytherapy for the treatment of early-stage breast carcinoma. Cancer. 2006;106(5):991-999.

48. Kuske RR, Winter K, Arthur DW, et al. Phase II trial of brachytherapy alone after lumpectomy for select breast cancer: toxicity analysis of RTOG 95-17. Int J Radiat Oncol Biol Phys. 2006;65(1): 45-51.

49. Arthur DW, Winter K, Kuske RR, et al. A Phase II trial of brachytherapy alone after lumpectomy for select breast cancer: tumor control and survival outcomes of RTOG 95-17. Int J Radiat Oncol Biol Phys. 2008;72(2):467-473.
50. Dickler A, Ivanov O, Francescatti D. Intraoperative radiation therapy in the treatment of early-stage breast cancer utilizing xoft axxent electronic brachytherapy. World J Surg Oncol. 2009;7:24.

51. Dickler A. Xoft Axxent electronic brachytherapy: a new device for delivering brachytherapy to the breast. Nat Clin Pract Oncol. 2009; 6(3): $138-142$

52. White SA, Landry G, Fonseca GP, et al. Comparison of TG-43 and TG-186 in breast irradiation using a low energy electronic brachytherapy source. Med Phys. 2014;41(6):061701.

53. Grobmyer SR, Lightsey JL, Bryant CM, et al. Low-kilovoltage, singledose intraoperative radiation therapy for breast cancer: results and impact on a multidisciplinary breast cancer program. J Am Coll Surg. 2013;216(4):617-623; discussion 623-614.

54. Vaidya JS, Joseph DJ, Tobias JS, et al. Targeted intraoperative radiotherapy versus whole breast radiotherapy for breast cancer (TARGIT-A trial): an international, prospective, randomised, non-inferiority phase 3 trial. Lancet. 2010;376(9735):91-102.

55. Jones R, Libby B, Showalter SL, et al. Dosimetric comparison of (192)Ir high-dose-rate brachytherapy vs $50 \mathrm{kV} \mathrm{X}$-rays as techniques for breast intraoperative radiation therapy: Conceptual development of image-guided intraoperative brachytherapy using a multilumen balloon applicator and in-room CT imaging. Brachytherapy. 2014;13(5): 502-507.

56. Beal K, McCormick B, Zelefsky MJ, et al. Single-fraction intraoperative radiotherapy for breast cancer: early cosmetic results. Int J Radiat Oncol Biol Phys. 2007;69(1):19-24.

57. Veronesi U, Gatti G, Luini A, et al. Intraoperative radiation therapy for breast cancer: technical notes. Breast J. 2003;9(2):106-112.

58. Arthur DW, Vicini FA, Todor DA, Julian TB, Cuttino LW, Mukhopadhyay ND. Contura Multi-Lumen Balloon breast brachytherapy catheter: comparative dosimetric findings of a phase 4 trial. Int $J$ Radiat Oncol Biol Phys. 2013;86(2):264-269.

59. Eyre K, Whitney D, Mukesh M, Wilson C, Coles C. Optimization and comparison of balloon-based partial breast brachytherapy using a single source, a standard plan line source, and both forward and inverse planned multilumen techniques. Brachytherapy. 2013;12(2):107-113.

60. Manoharan SR, Rodriguez RR, Bobba VS, Chandrashekar M. Dosimetry evaluation of SAVI-based HDR brachytherapy for partial breast irradiation. J Med Phys. 2010;35(3):131-136.

61. Scanderbeg DJ, Yashar C, Rice R, Pawlicki T. Clinical implementation of a new HDR brachytherapy device for partial breast irradiation. Radiother Oncol. 2009;90(1):36-42.

62. Yashar CM, Blair S, Wallace A, Scanderbeg D. Initial clinical experience with the Strut-Adjusted Volume Implant brachytherapy applicator for accelerated partial breast irradiation. Brachytherapy. 2009;8(4):367-372.

63. Yashar CM, Scanderbeg D, Kuske R, et al. Initial clinical experience with the Strut-Adjusted Volume Implant (SAVI) breast brachytherapy device for accelerated partial-breast irradiation (APBI): first 100 patients with more than 1 year of follow-up. Int J Radiat Oncol Biol Phys. 2011;80(3):765-770.

64. Dickler A, Seif N, Kirk MC, et al. A dosimetric comparison of MammoSite and ClearPath high-dose-rate breast brachytherapy devices. Brachytherapy. 2009;8(1):14-18.

65. Njeh CF, Saunders MW, Langton CM. Accelerated Partial Breast Irradiation (APBI): a review of available techniques. Radiat Oncol. 2010;5:90.

66. Rivard MJ, Melhus CS, Wazer DE, Bricault RJ Jr. Dosimetric characterization of round HDR 192Ir AccuBoost applicators for breast brachytherapy. Med Phys. 2009;36(11):5027-5032.

67. Hamid S, Rocchio K, Arthur D, et al. A multi-institutional study of feasibility, implementation, and early clinical results with noninvasive breast brachytherapy for tumor bed boost. Int J Radiat Oncol Biol Phys. 2012;83(5):1374-1380.

68. Leonard KL, Hepel JT, Styczynski JR, Hiatt JR, Dipetrillo TA, Wazer DE. Breast boost using noninvasive image-guided breast brachytherapy vs external beam: a 2:1 matched-pair analysis. Clin Breast Cancer. 2013;13(6):455-459. 
69. Hepel JT, Hiatt JR, Sha S, et al. The rationale, technique, and feasibility of partial breast irradiation using noninvasive image-guided breast brachytherapy. Brachytherapy. 2014;13(5):493-501.

70. Tuschy B, Berlit S, Nasterlack C, et al. Intraoperative radiotherapy of early breast cancer using low-kilovoltage x-rays-reasons for omission of planned intraoperative irradiation. Breast J. 2013;19(3):325-328.

71. Zannis V, Beitsch P, Vicini F, et al. Descriptions and outcomes of insertion techniques of a breast brachytherapy balloon catheter in 1403 patients enrolled in the American Society of Breast Surgeons MammoSite breast brachytherapy registry trial. Am J Surg. 2005; 190(4):530-538.

72. Koh VY, Buhari SA, Tan PW, et al. Comparing a volume based template approach and ultrasound guided freehand approach in multicatheter interstitial accelerated partial breast irradiation. J Contemp Brachytherapy. 2014;6(2):173-177.

73. DeBiose DA, Horwitz EM, Martinez AA, et al. The use of ultrasonography in the localization of the lumpectomy cavity for interstitial brachytherapy of the breast. Int J Radiat Oncol Biol Phys. 1997; 38(4):755-759.

74. Cuttino LW, Todor D, Arthur DW. CT-guided multi-catheter insertion technique for partial breast brachytherapy: reliable target coverage and dose homogeneity. Brachytherapy. 2005;4(1):10-17.

75. Polgar C, Major T, Somogyi A, et al. [CT-image-based conformal brachytherapy of breast cancer. The significance of semi-3-D and 3-D treatment planning]. Strahlenther Onkol. 2000;176(3):118-124.

76. Aristei C, Tarducci R, Palumbo I, et al. Computed tomography for excision cavity localization and 3D-treatment planning in partial breast irradiation with high-dose-rate interstitial brachytherapy. Radiother Oncol. 2009;90(1):43-47.

77. Mutyala S, Yaparpalvi R, Choi W, Mehta K, Spierer M, Kalnicki S Placement of MammoSite brachytherapy catheter under computedtomography scan guidance. Technol Cancer Res Treat. 2009; 8(3):177-180.

78. Jolicoeur M, Racine ML, Trop I, et al. Localization of the surgical bed using supine magnetic resonance and computed tomography scan fusion for planification of breast interstitial brachytherapy. Radiother Oncol. 2011;100(3):480-484.

79. Vaidya JS, Wenz F, Bulsara M, et al. Risk-adapted targeted intraoperative radiotherapy versus whole-breast radiotherapy for breast cancer: 5-year results for local control and overall survival from the TARGIT-A randomised trial. Lancet. 2014;383(9917):603-613.

80. Veronesi U, Orecchia R, Maisonneuve P, et al. Intraoperative radiotherapy versus external radiotherapy for early breast cancer (ELIOT): a randomised controlled equivalence trial. Lancet Oncol. 2013;14(13): 1269-1277.

81. Trifiletti DM, Jones R, Showalter SL, et al. Techniques for intraoperative radiation therapy for early-stage breast carcinoma. Future Oncol. 2015;11(7):1047-1058.

82. Orcutt KP, Libby B, Handsfield LL, Moyer G, Showalter TN. CT-onrails-guided HDR brachytherapy: single-room, rapid-workflow treatment delivery with integrated image guidance. Future Oncol. 2014; 10(4):569-575.

83. Vicini F, Kini VR, Chen P, et al. Irradiation of the tumor bed alone after lumpectomy in selected patients with early-stage breast cancer treated with breast conserving therapy. J Surg Oncol. 1999;70(1):33-40.
84. Krishnan L, Jewell WR, Tawfik OW, Krishnan EC. Breast conservation therapy with tumor bed irradiation alone in a selected group of patients with stage I breast cancer. Breast J. 2001;7(2):91-96.

85. Perera F, Engel J, Holliday R, et al. Local resection and brachytherapy confined to the lumpectomy site for early breast cancer: a pilot study. J Surg Oncol. 1997;65(4):263-267; discussion 267-268.

86. Baglan KL, Martinez AA, Frazier RC, et al. The use of high-dose-rate brachytherapy alone after lumpectomy in patients with early-stage breast cancer treated with breast-conserving therapy. Int J Radiat Oncol Biol Phys. 2001;50(4):1003-1011.

87. Krishnan L, Krishnan EC, Wolf CD, Jewell WR. Preservation of augmented breasts in patients with breast cancer. Radiographics. 1993; 13(4):831-839.

88. Kuske R. Breast conservation therapy without capsular contracture in young augmented women using interstitial brachytherapy. J Contemp Brachytherapy. 2014;6(2):231-235.

89. Bloom ES, Kirsner S, Mason BE, et al. Accelerated partial breast irradiation using the strut-adjusted volume implant single-entry hybrid catheter in brachytherapy for breast cancer in the setting of breast augmentation. Brachytherapy. 2011;10(3):178-183.

90. Akhtari M, Nitsch PL, Bass BL, Teh BS. Long-term outcome of accelerated partial breast irradiation using a multilumen balloon applicator in a patient with existing breast implants. Brachytherapy. 2015; 14(2):289-292.

91. Roth AM, Kauer-Dorner D, Resch A, et al. Is oncoplastic surgery a contraindication for accelerated partial breast radiation using the interstitial multicatheter brachytherapy method? Brachytherapy. 2014;13(4):394-399.

92. Kim Y, Arshoun Y, Trombetta MG. Pacemaker/implantable cardioverter-defibrillator dose in balloon high-dose-rate brachytherapy for breast cancer treatment. Brachytherapy. 2012;11(5):380-386.

93. Croshaw R, Kim Y, Lappinen E, Julian T, Trombetta M. Avoiding mastectomy: accelerated partial breast irradiation for breast cancer patients with pacemakers or defibrillators. Ann Surg Oncol. 2011;18(12):3500-3505.

94. Sung W, Kim S, Kim JI, et al. Dosimetric perturbations due to an implanted cardiac pacemaker in MammoSite $\left({ }^{\circledR}\right)$ treatment. Med Phys 2012;39(10):6185-6191.

95. Arthur DW. RTOG 1014: a phase II study of repeat breast preserving surgery and 3D-Conformal Partial Breast Re-Irradiation (PBRI) for local recurrence of breast carcinoma [database on the Internet]. Available from: http://www.rtog.org/ClinicalTrials/ProtocolTable/StudyDetails. aspx?study=1014. Accessed April 10, 2015.

96. Chadha M, Feldman S, Boolbol S, Wang L, Harrison LB. The feasibility of a second lumpectomy and breast brachytherapy for localized cancer in a breast previously treated with lumpectomy and radiation therapy for breast cancer. Brachytherapy. 2008;7(1):22-28.

97. Resch A, Fellner C, Mock U, et al. Locally recurrent breast cancer: pulse dose rate brachytherapy for repeat irradiation following lumpectomy - a second chance to preserve the breast. Radiology. 2002;225(3): $713-718$.

98. Hannoun-Levi JM, Resch A, Gal J, et al. Accelerated partial breast irradiation with interstitial brachytherapy as second conservative treatment for ipsilateral breast tumour recurrence: multicentric study of the GEC-ESTRO Breast Cancer Working Group. Radiother Oncol. 2013;108(2):226-231.
Breast Cancer: Targets and Therapy

\section{Publish your work in this journal}

Breast Cancer: Targets and Therapy is an international, peerreviewed open access journal focusing on breast cancer research, identification of therapeutic targets and the optimal use of preventative and integrated treatment interventions to achieve improved outcomes, enhanced survival and quality of life for the cancer patient.

\section{Dovepress}

View the full aims and scopes of this journal here. The manuscript management system is completely online and includes a very quick and fair peer-review system, which is all easy to use. Visit http:// www.dovepress.com/testimonials.php to read real quotes from published authors. 